\title{
Central Venous Catheter-Related Infection in Critically Ill Patients
}

\section{العدوى ذات الصلة بالقسطرة الوريدية في المرضى ذوي الحالات الحرجة}

Dear Editor,

We read with great interest recent studies about infections related to central venous catheter (CVC) and associated risk factors in critically ill postoperative patients evaluated in Iran, Oman and Egypt. ${ }^{1-4}$ Beigmohammadi evaluated 66 Iranian patients who were in the intensive care unit (ICU) following cancer surgery. ${ }^{1}$ He reported 23 simple CVCs without antimicrobials and 43 CVCs impregnated with antimicrobial agents-with 28 impregnated internally and 15 impregnated internally and externally. ${ }^{1}$ The jugular (51.5\%) and subclavian (48.5\%) veins were the sites for insertion of the catheters. CVC-related infection was confirmed by positive cultures of blood samples from the catheters. A total of $22.7 \%$ of cases had positive blood cultures and $9.1 \%$ had catheter-related bloodstream infections; therefore, there were 5.8 infections per 1,000 catheter-days for CVCs. ${ }^{1}$ There were no significant differences between simple and impregnated CVCs with respect to catheter tip colonisation, as well as in relation to the prevention of bloodstream infections. ${ }^{1} \mathrm{Hamid}$ et al. evaluated the early and late complications of 204 doublelumen cuffed tunnelled CVCs placed in 161 patients in a tertiary healthcare centre in Oman. ${ }^{2}$ The jugular (90.6\%) and femoral (9.4\%) veins were the sites for insertion of the catheters and infections were detected in 29 catheters (22\%) in 22 patients. Staphylococcus aureus (55.2\%), acinetobacter and pseudomonas (20.6\% each) were the main infectious agents. A total of 18 (62.1\%) cases had a haematogenous origin, four (13.8\%) were exit site infections, and seven (24.1\%) were both. Most infected catheters had to be removed and few infections improved with antibiotics. ${ }^{2}$ Khalil and Azqul's prospective study in Egypt included 111 cardiac patients with CVCs. They found 11 (9.9\%) cases of infection by the following agents: coagulase negative staphylococci, acinetobacter, Alcaligenes faecalis, enterobacter and klebsiella. The compliance of health workers to CVC care recommendations played a role in the presence of infections. ${ }^{3}$

Al-Hadi and Sallam discussed additional complications of fractured CVCs lodged in the heart or in the pulmonary artery that may cause thrombosis, perforation, endocarditis or sepsis. ${ }^{4}$ They reported three patients: 1) a 45-year-old male patient with chronic renal failure under haemodialysis with a fractured catheter lodged in the right pulmonary artery; 2) a 55-year-old female patient with chronic renal failure under haemodialysis and a fractured catheter lodged in the left pulmonary artery; and 3) a 62-year-old male patient who had acute myocardial infarction and heart failure one month prior to his admission and a broken catheter in the right atrium. However, the short time from the catheter rupture to its retrieval avoided the occurrence of infection. ${ }^{4}$

Falcão Pedrosa Costa et al. reported a 40-year-old Brazilian female patient who had endocarditis from Burkholderia cepacia associated with an intra-cardiac fragment of a peripherally inserted central catheter utilised a long time prior during postpartum septic shock treated in an ICU..$^{5}$ Although this infectious agent is not common in hospitalised patients, it may occur in immunocompromised individuals. The unsuspected foreign body, infected after immunosuppression for renal transplant, was incidentally found via routine investigation of infective foci in a renal transplant patient. The authors highlighted the importance of monitoring the heart for infections in haemodialytic patients and end-stage renal disease as well as the risks of blood infection by peripherally inserted CVCs utilised in the ICU. ${ }^{5}$ Fragments of CVCs may cause embolisms, arrhythmias or cardiac perforation. ${ }^{5}$ The articles discussed herein emphasised that critically ill patients with cancer and end-stage renal disease are immunocompromised and more prone to severe infections. ${ }^{1,25,6}$ The main risk factors include ageing, haemodialysis, chemotherapy and the type of fluid infusion. ${ }^{1,2,5}$

Particular attention should be given to unspecific clinical manifestations and the growing number of gramnegative bacteria which often pose diagnostic challenges and may potentiate the mortality rate. ${ }^{1,3,5}$ Acinetobacter, burkholderia, klebsiella and enterobacter are microorganisms frequently identified among these groups of patients and their resistance to antibiotics is elevated. ${ }^{1-3,5}$ The two main concerns of such infections are the increasing mortality rate, which is up to $35 \%$, and increasing hospital costs. ${ }^{6}$ The infection routes begin at the tip and the 
internal lumen of the CVCs; therefore, routine prevention should involve better personnel training and more rigid procedures of sterilisation. ${ }^{1,3}$ Frasca et al. summarised the following main measures to reduce colonisation at the skin insertion site or the infusion line: 1) accurately following the protocols; 2) involvement of qualified personnel in catheter changing and care; 3) use of biomaterials that inhibit microorganism growth and adhesion; 4) good hand hygiene; 5) use of an alcoholic formulation of chlorhexidine for skin disinfection and manipulation of the vascular line; 6) choice of the subclavian vein route for insertion of CVCs using full-barrier care; and 7) removal of unnecessary catheters. ${ }^{6}$ The duration of catheterisation and the frequent catheter hub access have a large impact on the risks of colonisation in catheter-related infections, with migration of agents along the lumen of the catheter. ${ }^{6}$ Further research is needed to optimise the prevention and reduce the rate of CVC-related infections in immunocompromised hosts who are managed in an ICU environment. ${ }^{1,2}$ This letter aimed to enhance the suspicion index of non-specialised healthcare workers about a scarcely reported entity which may evolve unsuspectedly and has a poor patient outcome.

\section{"Vitorino M. dos Santos ${ }^{1}$ and Lister A. M. dos Santos ${ }^{2}$}

${ }^{1}$ Department of Internal Medicine, Armed Forces Hospital and Catholic University, Brasilia, Brazil, ${ }^{2}$ Department of General and Oncologic Surgery, State Workers Hospital, São Paulo, Brazil

"Corresponding Author's e-mail: vitorinomodesto@gmail.com

\section{References}

1. Beigmohammadi MT. Incidence of central venous catheter-related infection and risk factors in critically ill post-operative cancer patients: A randomized prospective cohort study. Arch Crit Care Med 2016; 1:e7732. https://doi.org/10.17795/accm-7732.

2. Hamid RS, Kakaria AK, Khan SA, Mohammed S, Al-Sukaiti R, Al-Riyami D, et al. Safety and complications of double-lumen tunnelled cuffed central venous dialysis catheters: Clinical and radiological perspective from a tertiary centre in Oman. Sultan Qaboos Univ Med J 2015; 15:e501-6. https://doi.org/10.18295/squmj.2015.15.04.010.

3. Khalil GM, Azqul MM. Risk factors and microbial profile of central venous catheter related blood stream infection in medical cardiac care units, National Heart Institute, Egypt. Egypt Heart J 2018; 70:361-4. https://doi.org/10.1016/j.ehj.2018.07.001.

4. Al-Hadi H, Sallam M. Retrieval of dislodged central venous pressure catheters: Early experience with three case presentations. Sultan Qaboos Univ Med J 2009; 9:175-9.

5. Falcão Pedrosa Costa A, Castelo Branco Cavalcanti F, Modesto dos Santos V. Endocarditis due to Burkholderia cepacia and an intracardiac foreign body in a renal transplant patient. Rev Port Cardiol 2014; 33:117.e1-4. https://doi.org/10.1016/j.repc.2013.09.012.

6. Frasca D, Dahyot-Fizelier C, Mimoz O. Prevention of central venous catheter-related infection in the intensive care unit. Crit Care 2010; 14:212. https://doi.org/10.1186/cc8853. 